\title{
The status and high risk factors of severe psychological distress in migraine patients during nCOV-2019 outbreak in Southwest China: a cross-sectional study
}

Mengmeng $\mathrm{Ma}^{\dagger}$, Jinghuan Fang ${ }^{\dagger}$, Changling Li, Jiajia Bao, Yang Zhang, Ning Chen, Jian Guo ${ }^{*}$ and Li He*

\begin{abstract}
Background: Psychological distress is highly prevalent among migraineurs during public health emergencies. The coronavirus disease 2019 (nCOV-2019) has created mass panic in China due to its highly contagious by contact and aerosols and lack of effective treatment. However, the emotion status of migraineurs stayed unclear during the nCOV-2019 outbreak.

Objective: To understand psychological distress of migraineurs by comparing with common population and identify potential high-risk factors of severe psychological distress among migraine patients.

Method: We enrolled the migraineurs treated at the department of Neurology of West China Hospital and healthy controls with age- and sex-matched to migraineurs. Data on clinicodemographics and psychological distress in the month of February 2020 (during in the nCOV-2019 outbreak in China) were collected. We used the Kessler 6-item (K-6) scale to assess psychological distress. Potential risk factors of severe psychological distress were identified using univariate and multivariate logistic regression.

Results: The 144 migraineurs and 150 controls were included in the study. Migraineurs showed significantly higher K-6 scores than controls $(P<0.001)$. Migraine attack frequency in previous 30 days and time spent paying attention to outbreak showed significant in multivariate logistic regression with respective odds ratios of $2.225(95 \% \mathrm{Cl} 1.361-$ $3.628, P=0.001)$ and $1.589(95 \% 1.117-2.26, P=0.01)$.
\end{abstract}

Conclusion: During public health outbreaks, healthcare professionals should focus not only on controlling and reducing migraine attack but also on mental health of migraineurs, especially those with high frequency migraine attack.

Keywords: COVID-19, Migraine, Severe psychological distress, Kessler 6-item psychological distress scale

\footnotetext{
*Correspondence: water-deep1983@163.com; heli2003new@126.com ${ }^{\dagger}$ Mengmeng Ma and Jinghuan Fang contributed equally to this work. Department of Neurology, West China Hospital, Sichuan University, Chengdu, China
}

C C The Author(s). 2020 Open Access This article is licensed under a Creative Commons Attribution 4.0 International License, which permits use, sharing, adaptation, distribution and reproduction in any medium or format, as long as you give appropriate credit to the original author(s) and the source, provide a link to the Creative Commons licence, and indicate if changes were made. The images or other third party material in this article are included in the article's Creative Commons licence, unless indicated otherwise in a credit line to the material. If material is not included in the article's Creative Commons licence and your intended use is not permitted by statutory regulation or exceeds the permitted use, you will need to obtain permission directly from the copyright holder. To view a copy of this licence, visit http://creativecommons.org/licenses/by/4.0/ The Creative Commons Public Domain Dedication waiver (http://creativecommons.org/publicdomain/zero/1.0/) applies to the data made available in this article, unless otherwise stated in a credit line to the data. 


\section{Background}

Migraine is a prevalent and disabling neurological disorder that is characterized by recurrent headache, sensitivity to light, nausea, and reduced functioning [1, 2]. Migraine affects 1 out of every 10 individuals among the whole world [3]. In the Global Burden of Disease Study 2017(GBD2017), migraine was ranked 7th based upon years lived with disability (YLDs) and ranked 2nd in the global burden of neurological disorders based on YLDs, which was higher than epilepsy and Alzheimer's disease $[4,5]$. Moreover, migraine was affected by several risk factors, especially emotion factors, such as anxiety and panic [6-8]. Anxiety and panic may increase migraine attack which increase migraineurs' economic burden [1, 6-8]. Therefore, it is necessary to know the emotion status of migraineurs and study the factors which may affect emotion status of migraineurs.

Since coronavirus disease 2019 (nCOV-2019) was first reported on 08 December 2019 in Wuhan, China [9], it is caused by a novel coronavirus, named COVID-19 or SARS-CoV-2 [10, 11]. nCOV-2019 has created mass panic in China and other countries due to its highly contagious by contact and aerosols and the lack of effective treatment. Globally, more than 3 million confirmed cases with nearly 200,000 deaths were caused by nCOV-2019 on Mar 11, 2020 [12]. Now the outbreak of nCOV-2019 has spread around the whole world and defined as a major public health emergency $[13,14]$. Chinese citizens were asked to stay home quarantine during this nCOV2019 event. Therefore, they can only communicate and get information from media, while almost all the media were focus on nCOV-2019 outbreak. Although scientifically framed information can help people understand and prevent the developing situation of nCOV-2019 [15], excessive attention to media reports about public health emergency can increase risk of psychiatric disorder [16-19]. Anxiety and panic in the public can be created by the flood of disaster-related information, and the mental health of populations can be affected, even threatened by these circumstances, based on World Health Organization assessment [20]. However, the emotion status of migraineurs stayed unclear when facing public health emergency.

While anxiety and panic may increase migraine attack, then serious headache may increase psychiatric disorder in migraineurs $[6,7]$. Therefore, we propose our hypothesis that migraineurs would have more trouble controlling their emotion than common population when facing public health emergency. The K-6 scale is a selfrating scale created by Kessler and his colleagues for preliminary screening of whether respondents have serious psychological diseases. The Mandarin version was validated in the World Mental Health Survey 25 and shows good reliability and validity [21].

The present study compared the severity of psychological distress between migraineurs and common population during the nCOV-2019 outbreak in southwest China by using Kessler 6-item psychological distress scale and a custom-designed questionnaire. It also explored risk factors for severe psychological distress among migraineurs, which showed the frequency of migraine attacks and attention paid to media coverage of the nCOV-2019 outbreak might cause severe psychological distress in migraineurs.

\section{Method}

This is a cross-sectional case control study. Respondents were asked the questionnaire information about the month of February 2020, most into the COVID-19 outbreak in China.

\section{Study participants}

All the patients were recruited from the Department of Neurology of West China Hospital. All the participants were 18-60 years of age and have been diagnosed with migraine without aura at least 1 year before, the treatment of migraine was unchanged and did not take any other drugs regularly for 3 months at least. Moreover they had to be followed up monthly by a neurology specialist. Patients were excluded if they were confirmed COVID-19; or they were diagnosed with other neurological or psychiatric disorders; or had a history of alcohol or drug abuse, or uncontrolled psychosis; or they were unable to read or understand the questionnaires. The diagnosis of migraine without aura was made according to the International Classification of Headache Disorders, 3rd edition (ICHD-3).

In parallel, we invited a control group of healthy visitors of patients who were unrelated to those patients. Healthy controls were age- and sex-matched to migraine patients. The healthy controls had no history of migraine or other types of headache, psychiatric disorders, and alcohol or drug abuse and did not take any other drugs regularly for 3 months at least also.

The study protocol was approved by the Local Ethics Committee of West China Hospital, Sichuan University. The participants gave informed consent.

\section{Survey instruments}

Enrolled participants were sent on-line questionnaires via WeChat and were asked to fill them out.

\section{Questionnaire on demographics, clinical data, epidemiological contact information}

The questionnaire for this study included the demographics information about sex, age, marriage status, 
education level; clinical data about migraine information (attack frequency), medical history (including mental illness history, and treatment history) from electronic clinical records in the West China Hospital outpatient department; and epidemiological contact information about their level of concern over the COVID-19 outbreak (on a 4-point scale from "relatively unconcerned or very unconcerned" to "very concerned"), and the time they spent each day receiving information about the outbreak.

\section{Kessler 6-item psychological distress scale (K-6)}

The K-6 scale is a self-rating scale created by Kessler and his colleagues for preliminary screening of whether respondents have serious psychological diseases. It has been validated to assess non-specific psychological distress during the past 30 days [22], and it is often used in large-scale investigations [23] . The Mandarin version was validated in the World Mental Health Survey 25 and shows good reliability and validity [21]. The scale includes 6 psychological symptoms: "nervous", "hopeless", "restless or fidgety", "so depressed that nothing could cheer you up", "Everything is an effort" and "worthless" [22]. The Link 5 score is adopted, " 0 " means no time, " 1 " means occasionally, " 2 " part of time, " 3 " means most of time, and "4" means all the time, with a total score of 0 $\sim 24$ [24]. Severe psychological distress was defined as a total score $>12$ [21].

\section{Statistical analysis}

Statistical analysis was performed using SPSS 22.0 (IBM, Chicago, IL, USA). The values are reported as absolute numbers with percentages for categorical variables and as means with SDs for continuous variables. $\mathrm{X}^{2}$ tests and $\mathrm{t}$-tests were performed to compare categorical variables between groups, continuous variables between two groups respectively. K-6 scores showed a skewed distribution, so the rank sum test was used to compare the two groups. Univariate logistic regression was used to determine the association between clinicodemographic factors and severe psychological distress. Multivariate logistic regression was used to explore factors independently associated with severe psychological distress in patients with migraine. The multivariate model contained variables that were associated with $p<0.1$ in univariate analysis.

The level of statistical significance was defined as combined $P<0.05$.

\section{Results}

Of the 160 patients with migraine participated in the survey, 7 were excluded because they had mental disease before, 8 because they had alcohol or drug abuse and 1 because the patients were confirmed case of COVID-19.
In the end, 144 migraine patients (90\%) were enrolled. Then we invited 150 healthy controls with age- and sexmatched to migraine patients.

\section{Demographic and clinical characteristics}

Subject demographics and clinical characteristics were shown in Table 1. Patients and healthy controls showed no significant differences in sex, age, marriage status, education level, history of epidemiological contact, suspected COVID-19 cases, COVID - 19 cases in respondent's residential community and feeling difficulty to control emotion during the nCOV-2019 outbreak. Compared to healthy controls, Patients reported significantly lower family income $(P<0.001)$. Besides, migraine patients reported significantly greater concern about the outbreak $(P<0.001)$ and more time paying attention to outbreak $(P<0.001)$ and higher frequency of dreaming about nCOV-2019 $(P=0.019)$ than healthy controls.

\section{K-6 scores}

The K-6 scores were shown in Table 2. Compared with healthy controls, patients with migraine had significantly higher K-6 scores than healthy controls $(P<0.001)$, including for the items about feeling "nervous", "hopeless", "restless or fidgety", "so depressed that nothing could cheer you up", and "everything is an effort". In contrast, the two groups scored had no difference on the item about "feeling worthless". The proportion of patients with severe psychological distress, whose scores were more than $12(17 \%)$, was significantly greater than the controls group $(8 \%)(P=0.016)$.

\section{Assessment of the clinicodemographic factors associated with severe psychological distress}

The results of univariate and multivariate logistic regression were separately shown in Table 3 and Table 4 . Univariate analyses showed that the age $(P=0.033)$, migraine attack frequency in previous 30 days $(P=$ $0.005)$, history of epidemiological contact $(P=0.018)$, suspected COVID-19 cases $(P<0.001)$, COVID-19 cases in respondent's residential community $(P=0.004)$, time spent paying attention to outbreak $(P<0.001)$ and frequency of dreaming about nCOV-2019 $(P=0.044)$. Only migraine attack frequency in previous 30 days and time spent paying attention to outbreak showed significant in multivariate logistic regression with respective odds ratios of $2.23(95 \% \mathrm{CI} 1.36-3.63, P=0.001)$ and $1.59(95 \%$ $1.12-2.26, P=0.01)$.

\section{Discussion}

Migraineurs were more likely to have severe psychological distress than common population. After further explore risk factors related to severe psychological distress in migraineurs during nCOV-2019 outbreak, we 
Table 1 Clinicodemographic characteristics of participants

\begin{tabular}{|c|c|c|c|}
\hline characteristic & Migraine patients $(n=144)$ & Healthy controls $(n=150)$ & $P$ \\
\hline Male & $32(22 \%)$ & $39(26 \%)$ & 0.449 \\
\hline Age, year & $36.6 \pm 8.4$ & $36.8 \pm 9.5$ & 0.88 \\
\hline Marriage & $98(68 \%)$ & 119 (79\%) & 0.088 \\
\hline \multicolumn{4}{|l|}{ Education level, year } \\
\hline$\leq 12$ & $59(41 \%)$ & $50(33 \%)$ & \multirow[t]{2}{*}{0.175} \\
\hline$>12$ & $85(59 \%)$ & $100(67 \%)$ & \\
\hline \multicolumn{4}{|l|}{ Family monthly income ${ }^{\mathrm{a}}$, RMB } \\
\hline 0-4999 & $31(22 \%)$ & $37(25 \%)$ & \multirow[t]{7}{*}{$<0.001$} \\
\hline 5000-9999 & $41(29 \%)$ & $51(34 \%)$ & \\
\hline $10,000-14,999$ & $52(36 \%)$ & $34(23 \%)$ & \\
\hline $15,000-19,999$ & $12(8 \%)$ & $11(7 \%)$ & \\
\hline$>20,000$ & $8(6 \%)$ & $16(11 \%)$ & \\
\hline Duration of migraine, $y$ & $7.41 \pm 5.72$ & & \\
\hline Migraine attack frequency in previous 30 days & $3.58 \pm 3.28$ & & \\
\hline History of epidemiological contact & $4(3 \%)$ & $4(3 \%)$ & 0.953 \\
\hline Suspected COVID-19 cases & 21 & 12 & 0.074 \\
\hline COVID-19 cases in respondent's residential community & $23(26 \%)$ & $15(10 \%)$ & 0.127 \\
\hline \multicolumn{4}{|l|}{ Level of concern about 2019-nCoV outbreak } \\
\hline Very concerned & $95(66 \%)$ & $67(45 \%)$ & \multirow[t]{4}{*}{$<0.001$} \\
\hline Concerned & $32(22 \%)$ & $68(45 \%)$ & \\
\hline Average & $17(12 \%)$ & $12(8 \%)$ & \\
\hline Relatively unconcerned or very unconcerned & $0(0)$ & $3(2 \%)$ & \\
\hline Time spent paying attention to outbreak, hours per day & $2.3 \pm 1.5$ & $1.5 \pm 1.0$ & $<0.001$ \\
\hline Feeling difficulty to control emotion during the 2019-nCoV outbreak & $18(13 \%)$ & $11(7 \%)$ & 0.137 \\
\hline \multicolumn{4}{|l|}{ Frequency of dreaming about 2019-nCoV } \\
\hline most of time & $10(7 \%)$ & $5(3 \%)$ & \multirow[t]{3}{*}{0.019} \\
\hline sometimes & 28 (19\%) & 15 (10\%) & \\
\hline none & 106 (74\%) & 130 (87\%) & \\
\hline
\end{tabular}

Values are $\mathrm{n}(\%)$, mean $\pm \mathrm{SD}$, or median (maximum, minimum)

${ }^{a} 1 \mathrm{RMB}=0.143$ USD in 29 Feb 2020

Table 2 Scores of participants on the Kessler 6-item psychological distress scale

\begin{tabular}{llll}
\hline K6 item & Migraine patients & Healthy controls & $P$ \\
\hline Nervous & $1(4,0)$ & $0(4,0)$ & $0(4,0)$ \\
Hopeless & $1(3,0)$ & $0(3,0)$ & $<0.001$ \\
Restless or fidgety & $1(4,0)$ & $0(3,0)$ & $<0.001$ \\
So depressed that nothing could cheer you up & $1(4,0)$ & $0(2,0)$ & 0.001 \\
Everything is an effort & $1(2,0)$ & $0(2,0)$ & $<0.001$ \\
Worthless & $0(1,0)$ & $0(16,0)$ & 0.598 \\
Total score & $5(18,0)$ & $12(8 \%)$ & 0.001 \\
Severe psychological distress $(\mathrm{n}, \%)$ & $25(17 \%)$ & & 0.001
\end{tabular}

Values are median (maximum, minimum) or $\mathrm{n}(\%)$ 
Table 3 Exploration of factors associated with severe psychological distress (K6 score > 12) among migraine patients

\begin{tabular}{lll}
\hline Factor & Odds ratio $(95 \% \mathrm{Cl})$ & $P$ \\
\hline Sex & $1.62(0.51,5.10)$ & 0.414 \\
Age & $0.94(0.88,1.00)$ & 0.033 \\
Marriage & $0.87(0.40,1.88)$ & 0.718 \\
Education level & $1.29(0.53,3.15)$ & 0.794 \\
Family monthly income & $1.23(0.77,1.99)$ & 0.386 \\
Duration of migraine & $1.00(0.93,1.08)$ & 0.946 \\
Migraine attack frequency in previous 30 days & $7.67(1.83,32.12)$ & 0.005 \\
History of epidemiological contact & $16.09(1.6,161.86)$ & 0.018 \\
Suspected CovID-19 cases & $11.24(4,32.26)$ & $<0.001$ \\
CoVID-19 cases in respondent's & $4.22(1.57,11.36)$ & 0.004 \\
residential community & & 0.654 \\
Level of concern about 2019-nCoV outbreak & $1.16(0.61,2.23)$ & $<0.001$ \\
Time spent paying attention to outbreak, hours per day & $2.99(1.94,4.63)$ & 0.044 \\
Frequency of dreaming about 2019-nCoV & $1.91(1.02,3.59)$ & \\
\hline
\end{tabular}

Reference conditions for OR calculations: female, married, $\leq 12$ years of education, had history of epidemiological contact, had suspected COVID-19, had COVID-19 cases in respondent's residential community

found the frequency of migraine attacks and attention paid to media coverage of the nCOV-2019 outbreak is the 2 high risk factors for psychological distress in migraineurs.

\section{Compare to common population, migraineurs were more likely to have severe psychological distress during nCOV- 2019 outbreak}

On one hand, Psychological distress among migraineurs has significantly higher level of it than controls. The highest score in Kessler 6-item psychological distress scale of migraineurs is 18 , while it is 16 in common population. And the total score of Kessler 6-item psychological distress scale of migraineurs group were also higher than it of common population. On the other hand, there were $17 \%$ of migraineurs group and $8 \%$ of control group showed severe psychological distress. The percentage of severe psychological distress of both groups are significantly higher than it of the general population (3.97\%) in China before nCOV-2019 outbreak [21]. Our results matched the fact that public health emergency can increase risk of psychiatric disorder $[16,18,25]$. Moreover, the percentage of severe psychological distress of common population was also higher than the percentage of post-traumatic stress symptoms in China hardest-hit areas a month after the COVID-19 outbreak (7\%) [23]. This may cause by time gap. Liu [26] surveyed at about January, while we surveyed at February 1st. Compare to January, much more suspect and confirm cases of COVID-19 showed in February and Chinese citizens stayed home to for longer time, more media information flooded in [25]. Studies also showed that longer quarantine duration would cause psychological distress $[16,27]$. There is an increasing evidence that during public health emergency psychological distress together with alexithymia may be considered risk factors for negative outcomes (e.g., suicide), even simply increasing the risk of development of

Table 4 Multivariate logistic regression to identify independent predictors of severe psychological distress (K6 score $\geq 12$ )

\begin{tabular}{llll}
\hline Factor & OR & $95 \% \mathrm{Cl}$ & $P$ \\
\hline Age & 1.04 & $0.88,1.06$ \\
Migraine attack frequency in previous 30 days & 1.59 & $1.12,2.26$ & 0.428 \\
History of epidemiological contact & 17.31 & $0.32,94.66$ \\
Suspected COVID-19 cases & 3.56 & $0.77,16.39$ \\
COVID-19 cases in respondent's & 1.58 & $0.33,7.59$ \\
residential community & 2.23 & $1.36,3.63$ \\
Time spent paying attention to outbreak, hours per day & 1.38 & 0.163 \\
Frequency of dreaming about 2019-nCoV & $0.26,2.03$ \\
\hline
\end{tabular}

Reference conditions for OR calculations: had history of epidemiological contact, had suspected COVID-19, had COVID-19 cases in respondent's residential community 
depressive symptoms [28, 29]. In addition, the unique sensory processing patterns of individuals with major affective disorders and their relationship with psychiatric symptomatology have been clearly reported. Hyposensitivity or hypersensitivity may be "trait" markers of individuals with major affective disorders [30]. Taken together, it indicated that migraineurs may have more trouble controlling their emotions than common population.

\section{Factors related to severe psychological distress in migraineurs during nCOV-2019 outbreak}

The average hours spent daily on media coverage of the nCOV-2019 outbreak in migraineurs are $2.3 \mathrm{~h}$ compare to $1.5 \mathrm{~h}$ in common population. We found the amount of time spent daily on media coverage of the nCOV2019 outbreak were associated with severe psychological distress among migraineurs during the nCOV-2019 outbreak. This result matched the fact that higher social media exposure were associated with/increased the risk of psychological distress [16, 31, 32].

We also found that migraine attack frequency in previous 30 days was associated with severe psychological distress among migraineurs during the nCOV-2019 outbreak. It is well known that migraine attack caused migraineurs serious headache, which may cause increase anxiety emotion, such as fear of pain or another attack [7]. And facing nCOV-2019 outbreak was anxiety and panic emotion trigger $[16,25]$. Combine with these two factors, this result indicated that during public health emergency, migraineurs should take measures or at least pay more attention to control or reduce migraine attack and should focus on only the necessary media information about the outbreak in order to avoid severe psychological distress.

In addition, migraineurs who suffered from severe psychological distress should consult psychiatrist as soon as possible. It is a challenge for migraineurs to achieve treatment in time during this nCOV-2019 outbreak. Studies have recommended telepsychiatry, including online clinic, social media and the internet, to provide clinical resources and group emotional support for migraineurs [29].

\section{Limitations of the study}

First, we only collected data at south-western China, while currently the epidemic was widespread. However, potential relationships of psychological distress with distance between the epicenter of the epidemic should be taken considered, because such a relationship has been shown for earthquake survivors [33]. Second, psychological distress and time spent daily paying attention to the outbreak were based on self-report, which may increase risk of bias. Third, the details of media were not collected. The different type of media might affect the amount of information that responders got [32]. But this would not change out results. At last, we only include 144 migraineurs and 150 controls. Therefore, our results should be verified and extended in larger patient samples.

\section{Conclusions}

During public health outbreaks, healthcare professionals should focus not only on controlling migraine attack but also on mental health of migraineurs, especially those with high frequency migraine attack. They should advise migraineurs to avoid paying too much attention on media coverage of the outbreak.

\section{Abbreviations}

GBD2017: Global Burden of Disease Study 2017; YLDs: Years lived with disability; nCoV-2019: Coronavirus disease 2019; K-6: Kessler 6-item; ICHD-

3: The International Classification of Headache Disorders, 3rd edition

\section{Acknowledgments}

We thank the participants who volunteered for this study.

\section{Authors' contributions}

MMM and JHF were responsible for analyzing the data, drafting and revising the manuscript and they contributed equally to this work. CLL, JJB, YZ and NC were responsible for data acquisition and interpretation and drafting the manuscript. LH and JG designed the study and drafting the manuscript. All authors read and approved the final manuscript.

\section{Funding}

This project was supported by National Key R\&D Program of China (Nos. 2018 YFC1311400 and 2018YFC1311401); Fundamental Research Funds for the Central Universities (No. 2020SCU12032, the postdoctoral foundation of Sichuan University); "1.3.5 project for disciplines of excellence-Clinical Research Incubation Project, West China Hospital, Sichuan University" (No. 2018HXFH022).

\section{Availability of data and materials}

Not applicable. The datasets used and analyzed in the current study are not publicly available. Anonymous data is available from the corresponding author upon reasonable request.

\section{Ethics approval and consent to participate}

The study protocol was approved by the Local Ethics Committee of West China Hospital, Sichuan University. The participants gave informed consent.

\section{Consent for publication}

Consent to publish had been obtained from all the subjects.

\section{Competing interests}

The authors declare that they have no competing interests.

Received: 5 July 2020 Accepted: 3 August 2020

Published online: 12 August 2020

\section{References}

1. Jeyagurunathan A, Abdin E, Vaingankar JA et al (2020) Prevalence and comorbidity of migraine headache: results from the Singapore Mental Health Study 2016. Soc Psychiatry Psychiatr Epidemiol 55:33-43

2. Charles A (2018) The pathophysiology of migraine: implications for clinical management. Lancet Neurol 17:174-182

3. Woldeamanuel YW (2017) Headache in Resource-Limited Settings. Curr Pain Headache Rep 21:51

4. Feigin VL, Nichols E, Alam T et al (2019) Global, regional, and national burden of neurological disorders, 1990-2016: a systematic analysis for the Global Burden of Disease Study 2016. Lancet Neurol 18(5):459-480 
5. He L, Bao J, Ma M et al (2020) Early Age of Migraine Onset is Independently Related to Cognitive Decline and Symptoms of Anxiety Affect Quality of Life. Curr Neurovasc Res 17:1-11

6. Goulart AC, Santos IS, Brunoni AR et al (2014) Migraine headaches and mood/anxiety disorders in the ELSA Brazil. Headache 54:1310-1319

7. Smitherman TA, Kolivas ED, Bailey JR (2013) Panic disorder and migraine: comorbidity, mechanisms, and clinical implications. Headache 53:23-45

8. Ma M, Zhang J, Chen $\mathrm{N}$ et al (2018) Exploration of intrinsic brain activity in migraine with and without comorbid depression. J Headache Pain 19:48

9. Holshue ML, DeBolt C, Lindquist S et al (2020) First Case of 2019 Novel Coronavirus in the United States. N Engl J Med 382:929-936

10. Wang F-S, Zhang C (2020) What to do next to control the 2019-nCoV epidemic? Lancet 395:391-393

11. Chan KW, Wong VT, Tang SCW (2020) COVID-19: An Update on the Epidemiological, Clinical, Preventive and Therapeutic Evidence and Guidelines of Integrative Chinese-Western Medicine for the Management of 2019 Novel Coronavirus Disease. Am J Chin Med 48:737-762

12. Kaur N, Gupta I, Singh H et al (2020) Epidemiological and Clinical Characteristics of 6635 COVID-19 Patients: a Pooled Analysis. SN Compr Clin Med 382:727

13. Sanyaolu A, Okorie C, Marinkovic A et al (2020) Comorbidity and its Impact on Patients with COVID-19. SN Compr Clin Med 15:1

14. de SFLP, Valente TM, Tiraboschi FA et al (2020) Description of Covid-19 Cases in Brazil and Italy. SN Compr Clin Med:1-4

15. Zhao Y, Cheng S, Yu X et al (2020) Chinese Public's Attention to the COVID19 Epidemic on Social Media: Observational Descriptive Study. J Med Internet Res 22:e18825

16. Vindegaard N, Eriksen Benros M (2020) COVID-19 pandemic and mental health consequences: Systematic review of the current evidence. Brain Behav Immun

17. Lyons D, Frampton M, Naqvi S et al (2020) Fallout from the Covid-19 pandemic - should we prepare for a tsunami of post viral depression? Ir J Psychol Med:1-10

18. Rogers JP, Chesney E, Oliver D et al (2020) Psychiatric and neuropsychiatric presentations associated with severe coronavirus infections: a systematic review and meta-analysis with comparison to the COVID-19 pandemic. Lancet Psychiatry

19. Larsen SE, Lotfi S, Bennett KP et al (2019) A pilot randomized trial of a dua n-back emotional working memory training program for veterans with elevated PTSD symptoms. Psychiatry Res 275:261-268

20. Hao X, Zhou D, Li Z et al (2020) Severe psychological distress among patients with epilepsy during the COVID-19 outbreak in southwest China. Epilepsia

21. Y-k K, W-j G, Xu H et al (2015) The 6-item Kessler psychological distress scale to survey serious mental illness among Chinese undergraduates: Psychometric properties and prevalence estimate. Compr Psychiatry 63:105112

22. Kessler RC, Andrews G, Colpe LJ et al (2002) Short screening scales to monitor population prevalences and trends in non-specific psychological distress. Psychol Med 32:959-976

23. Kessler RC, Green JG, Gruber MJ et al (2010) Screening for serious mental illness in the general population with the K6 screening scale: results from the WHO World Mental Health (WMH) survey initiative. Int J Methods Psychiatr Res 19(Suppl 1):4-22

24. Wittchen $\mathrm{H}-\mathrm{U}$ (2010) Screening for serious mental illness: methodological studies of the K6 screening scale. Int J Methods Psychiatr Res 19(Suppl 1):13

25. Li S, Wang Y, Xue J et al (2020) The Impact of COVID-19 Epidemic Declaration on Psychological Consequences: A Study on Active Weibo Users. Int J Environ Res Public Health 17

26. Liu N, Zhang F, Wei C et al (2020) Prevalence and predictors of PTSS during COVID-19 outbreak in China hardest-hit areas: Gender differences matter. Psychiatry Res 287:112921

27. Brooks SK, Webster RK, Smith LE et al (2020) The psychological impact of quarantine and how to reduce it: rapid review of the evidence. Lancet 395: 912-920

28. de BD, Fornaro M, Orsolini L et al (2017) Alexithymia and Suicide Risk in Psychiatric Disorders: A Mini-Review. Front Psychiatry 8:148

29. Gautam M, Thakrar A, Akinyemi E et al (2020) Current and Future Challenges in the Delivery of Mental Healthcare during COVID-19. SN Compr Clin Med 2:865-870
30. Engel-Yeger B, Muzio C, Rinosi G et al (2016) Extreme sensory processing patterns and their relation with clinical conditions among individuals with major affective disorders. Psychiatry Res 236:112-118

31. Gao J, Zheng P, Jia Y et al (2020) Mental health problems and social media exposure during COVID-19 outbreak. PLoS ONE 15:e0231924

32. Chao M, Xue D, Liu T et al (2020) Media use and acute psychological outcomes during COVID-19 outbreak in China. J Anxiety Disord 74:102248

33. Kunii Y, Suzuki Y, Shiga T et al (2016) Severe Psychological Distress of Evacuees in Evacuation Zone Caused by the Fukushima Daiichi Nuclear Power Plant Accident: The Fukushima Health Management Survey. PLoS ONE 11:e0158821

\section{Publisher's Note}

Springer Nature remains neutral with regard to jurisdictional claims in published maps and institutional affiliations.
Ready to submit your research? Choose BMC and benefit from:

- fast, convenient online submission

- thorough peer review by experienced researchers in your field

- rapid publication on acceptance

- support for research data, including large and complex data types

- gold Open Access which fosters wider collaboration and increased citations

- maximum visibility for your research: over $100 \mathrm{M}$ website views per year

At BMC, research is always in progress.

Learn more biomedcentral.com/submissions 